\title{
In-line height profiling metrology sensor for zero defect production control
}

Rob Snel, Jasper Winters, Thomas Liebig, Wouter Jonker 


\title{
In-line height profiling metrology sensor for Zero Defect production control
}

\author{
Rob Snel, Jasper Winters, Thomas Liebig, Wouter Jonker, department of OptoMechatronics TNO, \\ Stieltjesweg 1, 2628CK Delft, The Netherlands
}

\begin{abstract}
Contemporary production systems of mechanical precision parts show challenges as increased complexity, tolerances shrinking to sub-microns and yield losses that must be mastered to the extreme. More advanced automation and process control is required to accomplish this task. Often a solution based on feedforward/feedback control is chosen requiring innovative and more advanced in line metrology. This article concentrates first on the context of in line metrology for process control and then on the development of a specific in line height profiling sensor. The novel sensor technology is based on full field time domain white light interferometry which is well know from the quality lab. The novel metrology system is to be mounted close to the production equipment, as required to minimize time delay in the control loop, and is thereby fully exposed to vibrations. This sensor is innovated to perform in line with an orders of magnitude faster throughput than laboratory instruments; it's robust to withstand the rigors of workshops and has a height resolution that is in the nanometer range.
\end{abstract}

Keywords: Zero Defect, advanced manufacturing, in-line metrology, sensors, height profiling, Industry4.0

\section{INTRODUCTION}

Manufacturing of millimeter sized mechanical precision parts encounter new challenges. The production manager is faced with Six-sigma manufacturing, first time right. This demands for more precise process control to meet the quality requirements while at the same time system throughput must be maintained and shorter setup times guaranteed in order to have economic relevance.

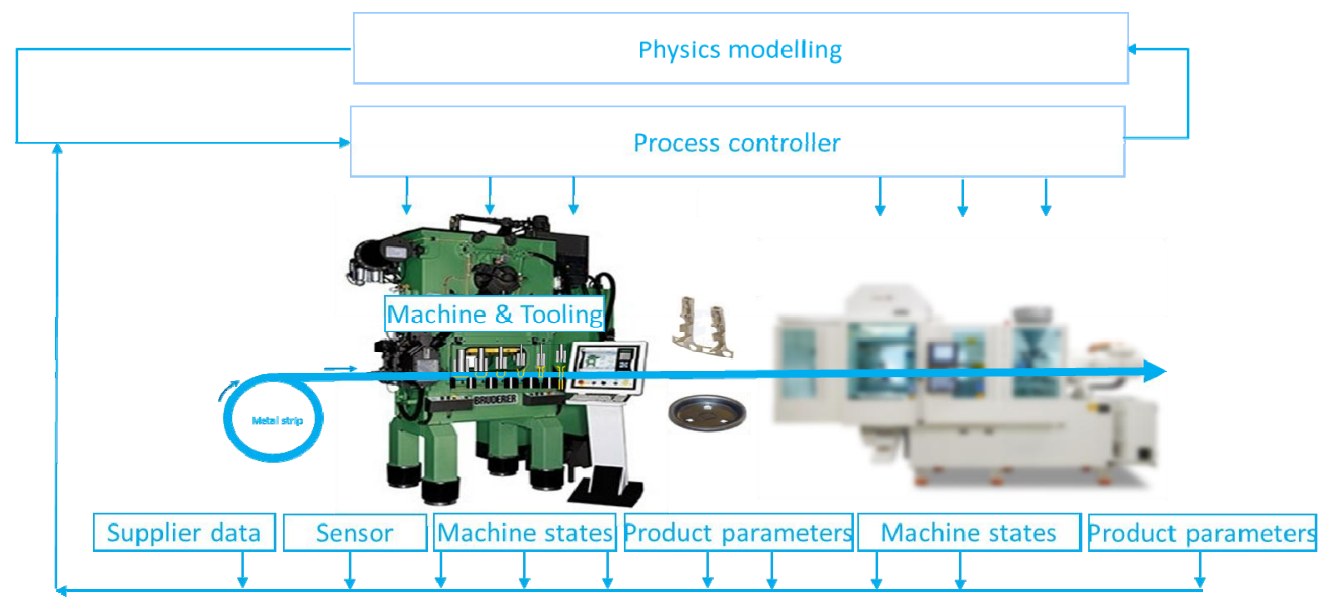

Figure 1 Digital twin with physics model based production line control

Optical Measurement Systems for Industrial Inspection X, edited by Peter Lehmann, Wolfgang Osten, Armando Albertazzi Gonçalves Jr., Proc. of SPIE Vol. 10329, 1032933 · (c) 2017 SPIE CCC code: $0277-786 \mathrm{X} / 17 / \$ 18 \cdot$ doi: $10.1117 / 12.2270711$ 
Actual production processes show the following trend:

- Increase of complexity combined with a reduction of product tolerances to sub-micron levels

- Yield loss must be mastered

- Inspection duration limits system throughput

For lean factories, this means future production machines are to be integrated in lines without buffers and the control of these machines is integrated into a control system on line level. Line level control is most likely a distributed asynchronous but real time state, process and product information acquisition system that is coupled to model based control: the digital twin. That model based control is likely to be a layering of (slow) model based parameter estimation physics models and (fast) parameter-execution process control loops (see Figure 1). One must distinguish between sensors for in line metrology and those for quality control for reasons of robustness to severe workshop conditions and timing considerations. Minimization of the signal delay between creation of the product and the corrective feedback to the cold forming press actuator is essential as to have a high control gain maximizing the intended disturbance reduction as defined in the overall Six-sigma production requirement. The in-line metrology system focused on in this article is required to operate in the fast loop of such a digital twin control scheme. Industry 4.0 fused with production physics.

\section{INDUSTRIAL END-USER REQUIREMENTS ANALYSIS}

Industrial end-users claim to have the need for an in-line height profiler with millimeter range and sub-micron resolution $>2 \mathrm{~Hz}$ repetition rate, $<0.25 \mathrm{~s}$ measurement duration; and very robust to industrial workshop conditions like. Close integration with the production process within centimeters distance of the production location. A more detailed requirements overview is stated in Table 1. Calibration to international standards. Network centric design for integration in ISA-95 information exchange topology. Flexibility to adjust the sensor system to different products and short production changeover adaptation time.

Table 1 Requirements table

\begin{tabular}{|c|c|c|c|}
\hline System requirement parameter & Required & Roadmap & \\
\hline Imaging Field of view & $>22$ & $>24$ & $\mathrm{~mm}$ \\
\hline Imaging Lateral resolution & $<75$ & $<25$ & $\mu \mathrm{m}$ \\
\hline Imaging Height range & $>5$ & $\ldots$ & $\mathrm{mm}$ \\
\hline Imaging Height accuracy $1 \sigma$ & $<750$ & $<10$ & $\mathrm{~nm}$ \\
\hline Imaging Height resolution $1 \sigma$ & $<100$ & $<5$ & $\mathrm{~nm}$ \\
\hline Imaging Capturing surface angle & $>45$ & $\cdots$ & $\circ$ \\
\hline Imaging Repetition rate & $>120$ & $>240$ & Products/minute \\
\hline Imaging Measurement duration & $<0.25$ & $\cdots$ & second \\
\hline Life time $24 / 7$ & $>7$ & $\cdots$ & years \\
\hline Operational temperature & $20+/-4$ & $\cdots$ & ${ }^{\circ} \mathrm{C}$ \\
\hline Humidity range & $0 \ldots 60$ & ... & $\mathrm{Rh} \%$ \\
\hline
\end{tabular}




\begin{tabular}{|l|l|l|l|}
\hline Ambient daylight spectrum & 500 & 1000 & $\mathrm{~W} / \mathrm{m} 2$ \\
\hline Incoming vibrations & See graph in Figure 2 \\
\hline & \\
\hline & $\begin{array}{l}\text { Tightest as possible integration with the production process. Intended for real time Six-sigma process } \\
\text { control on Critical To Quality product parameters }\end{array}$ \\
\hline
\end{tabular}

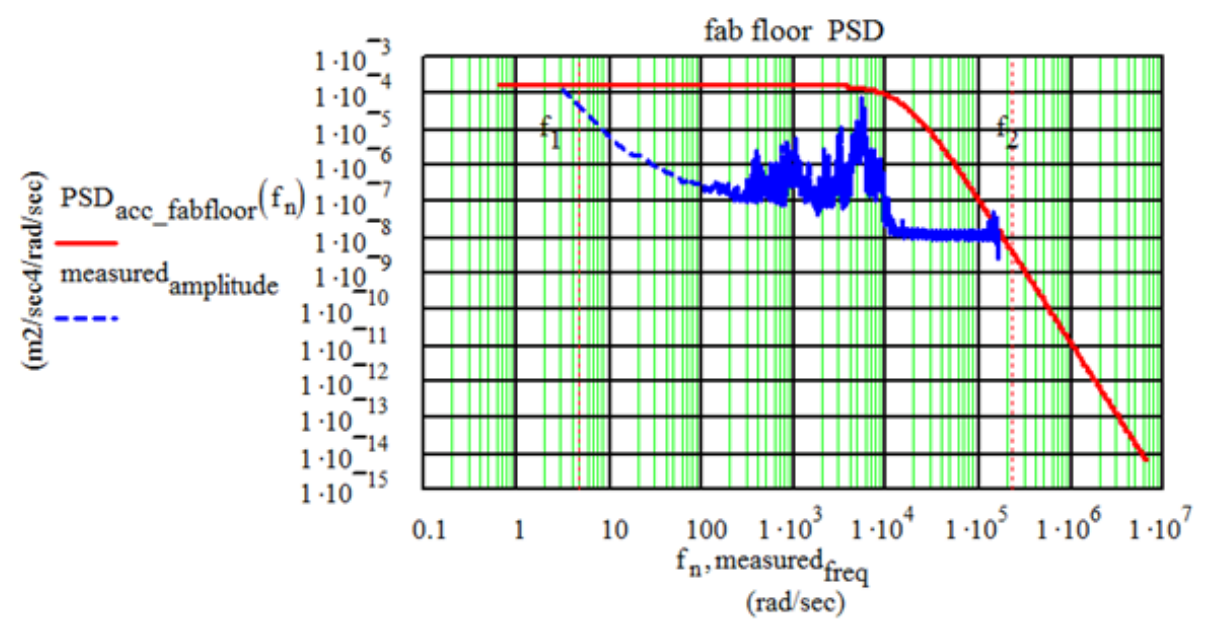

Figure 2 Typical workshop vibration as measured and design requirement

During the requirements analysis a number of technologies have been traded of against criteria as shown in table 2. A novel method of fast white light interferometry has been selected as most suitable to meet with current and future requirements. Beyond that, the technology allows for a parametric scalable design with a scalable measurement volume to adapt to production processes. 


\begin{tabular}{|c|c|c|c|c|c|c|c|c|}
\hline & 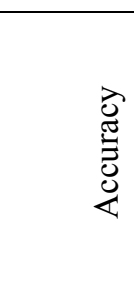 & $\begin{array}{l}\vec{D} \\
\mathbb{D} \\
\text { की }\end{array}$ & 感 & 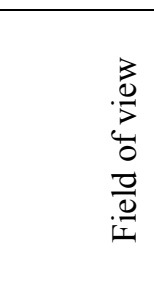 & 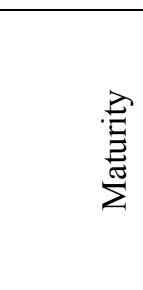 & 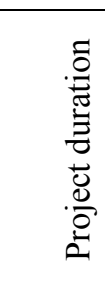 & 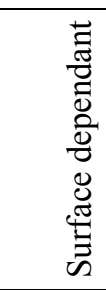 & 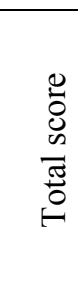 \\
\hline Technology & [750nm] & $\begin{array}{l}{[120} \\
\mathrm{rpm}]\end{array}$ & & {$[23 \mathrm{~mm}]$} & [COTS $]$ & {$[\mathrm{y} / \mathrm{n}]$} & {$[\mathrm{y} / \mathrm{n}]$} & \\
\hline Digital holography & 1 & 1 & -1 & 1 & -1 & 1 & 1 & 3 \\
\hline Interferometry & 0 & 0 & 1 & 1 & -1 & 1 & 0 & 2 \\
\hline Fringe projection & -1 & -1 & 1 & 1 & 0 & 1 & 0 & 1 \\
\hline Confocal & 1 & -1 & -1 & -1 & 1 & 1 & 1 & 1 \\
\hline X-ray & -1 & -1 & -2 & 1 & 1 & 1 & 1 & 0 \\
\hline $\begin{array}{l}\text { Laser angle/profile } \\
\text { measurement }\end{array}$ & -1 & 1 & 1 & -1 & 1 & 1 & -1 & 1 \\
\hline Fibre probe & 1 & -1 & 1 & 1 & -1 & -1 & 1 & 1 \\
\hline White light interferometry & 1 & -1 & -1 & 1 & 1 & 1 & 1 & 3 \\
\hline Light field photography & -1 & 1 & 1 & 1 & 1 & 1 & -1 & 3 \\
\hline $\begin{array}{l}\text { Fast white light } \\
\text { interferometry }\end{array}$ & 1 & 1 & 1 & 0 & 1 & 1 & 1 & 6 \\
\hline
\end{tabular}

\section{INSTRUMENT DESIGN}

The central element in the fast white light interferometer is a Michelson interferometer. See figure 3. Interferometric fringes are created by changing the length of the object-arm with respect to the reference arm. The mechatronic layout is a central optical axis that is aligned with the central thermal axis and motion systems have been optimized to a co-located design with reference sensors on the critical locations and carefully decoupled actuation in combination with air bearings. Closed loop motion control of the optics results in dynamic error suppression up to $150 \mathrm{Hertz}$. This enables direct mounting of the sensor on a cold forming press without isolation mounts.

The pixel-based quadrature interlock amplifiers perform amplitude demodulation up to $250 \times 10^{3}$ fringes per second, theoretically allowing for a maximum axial scan velocity of:

\section{$\lambda .250 \mathrm{KHz} / 4<35 \mathrm{~mm} / \mathrm{s}$}

Where $\lambda$ is the wavelength of the utilized excitation light source $(532 \mathrm{~nm})$. Balancing the noise figure resulted in $20 \mathrm{~mm} / \mathrm{s}$ as optimum. The benefit of the amplitude demodulation scheme is that it relaxes the AD-converter requirements and eliminate saturation of individual pixels due to ambient light. Large full well capacities of intermediate buffers allow for a high dynamic range, which enables a large angular capture range even for specular reflective surfaces or measurement on extreme black surfaces in a single stroke operation. Careful analysis of the error budget and error sources led to a system design which is a combination of optics and mechatronics that is capable to withstand these severe workshop circumstances and is still capable of delivering sub micrometer accuracy. 


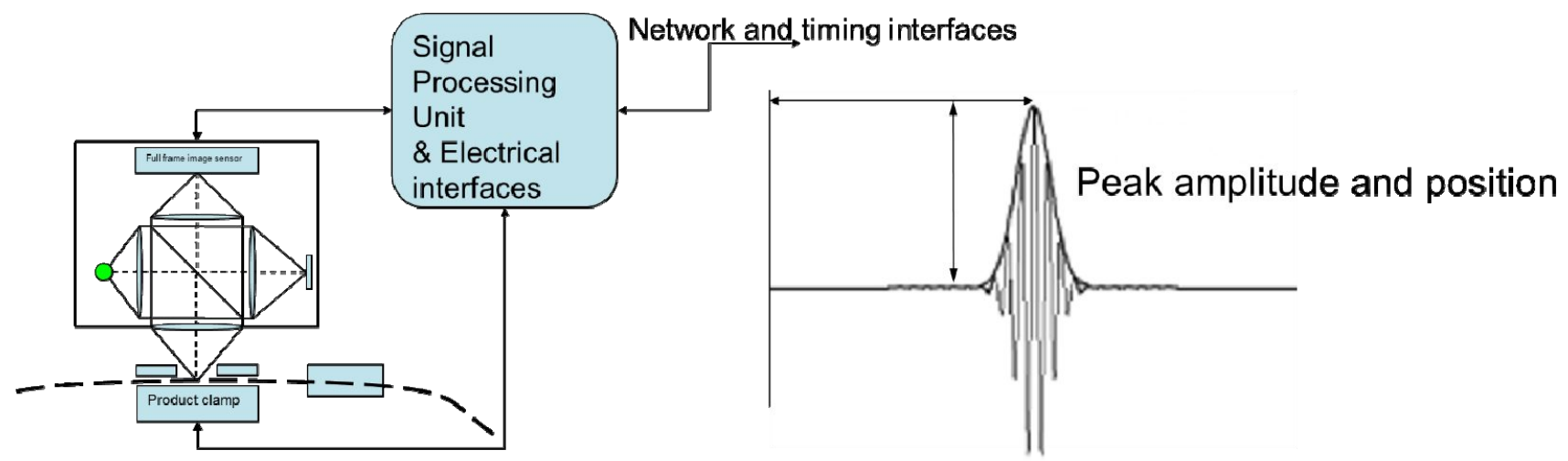

Figure 3 Principle system layout of the full frame time domain Michelson interferometer and interfaces

The sensor system design is optimized for

Total Uncertainty $=\operatorname{sqrt}\left(\mathrm{OS}_{\text {noise }}{ }^{2}+\mathrm{C}_{\text {noise }}{ }^{2}+\mathrm{M}_{\text {noise }}{ }^{2}+\mathrm{OI}_{\text {noise }}{ }^{2}\right)$

Where:

Transfer functions: (optics)

$\mathrm{OS}_{\text {noise }}=\mathrm{f}($ Image quality, optics stability)

$\mathrm{OI}_{\text {noise }}=\mathrm{f}($ Product, angle, pixel size $)$

Transfer functions: (camera)

$\mathrm{C}_{\text {noise }}=\mathrm{f}($ Signal, triggering $)$

Transfer functions: (mechatronics)

$\mathrm{M}_{\text {noise }}=\mathrm{f}($ Disturbances, Bandwidth, Parasitic motions $)$ 

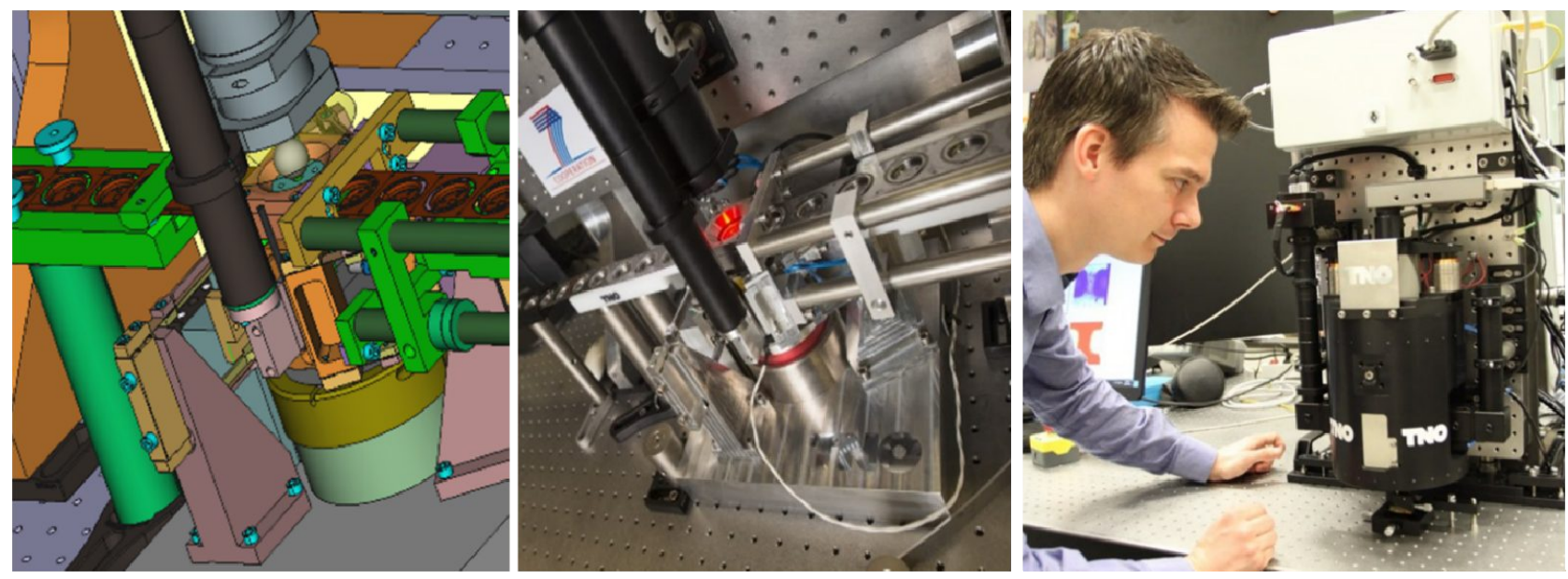

Figure 4 (left) 3D modeled design, (center) technology validation setup-TRL5, (right) technology demonstrator setup-TRL6

Signal processing utilizes a twinned set of a height- and signal strength images. Both height and signal strength are first calibrated and then pre-processed by means of data fusion to a sparse height map with reliable and accurate pixels only. Application depend processing to Critical To Quality parameters is performed thereafter. In case of the cold forming demonstration a total of 56 flatness- and outline-parameters are calculated in less than 0.2 second (Figure 5). From there on, model based control of the forming process is utilized to derive a meaningful corrective action to the actuator.
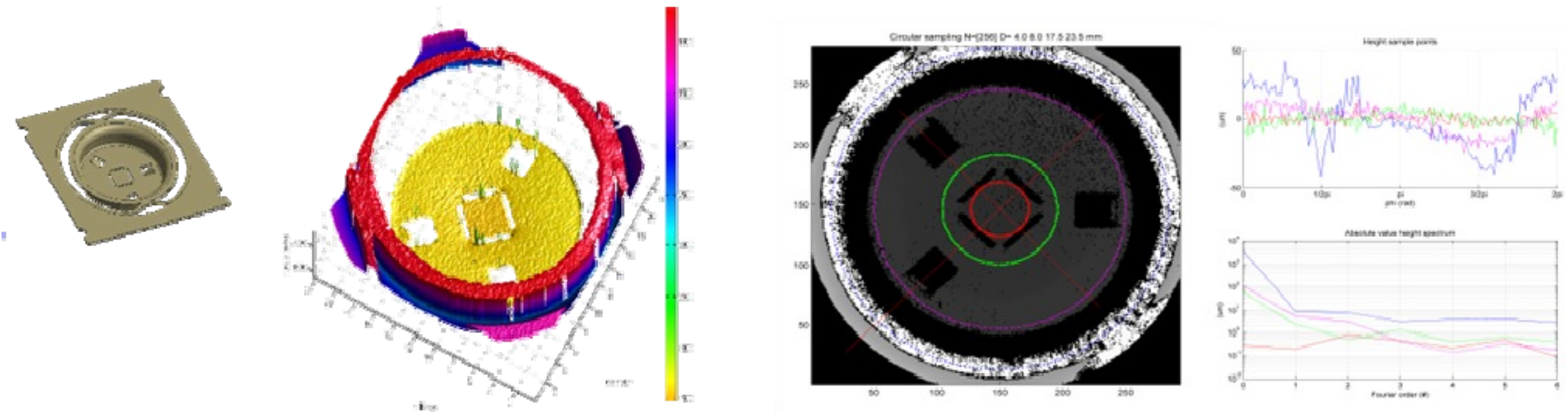

Figure 5 (left) product, (center) height profile, (right) real time derived Critical To Quality parameters 


\section{TESTING \& VERIFICATION METHOD}

Industrial reliability has been proven by a field test of 15 months in the cold forming line of a manufacturing workshop. During this field-test no modification or upgrades were performed. Upon retrieval to laboratory a Gage R\&R has been performed. This test had a duration of 57 minutes, during which 575 images where derived from the product. Gage R\&R repeatability test show a measurement system performance with a mean standard deviation of $1 \sigma=52 \mathrm{~nm}$ for the measurement instrument (see Figure 6). Different products where created with min-max experiments. Gage R\&R analysis shows that the measurement error is significant smaller than the product defects. Therefore the sensor is suitable to be used to qualify the products and control their manufacturing.
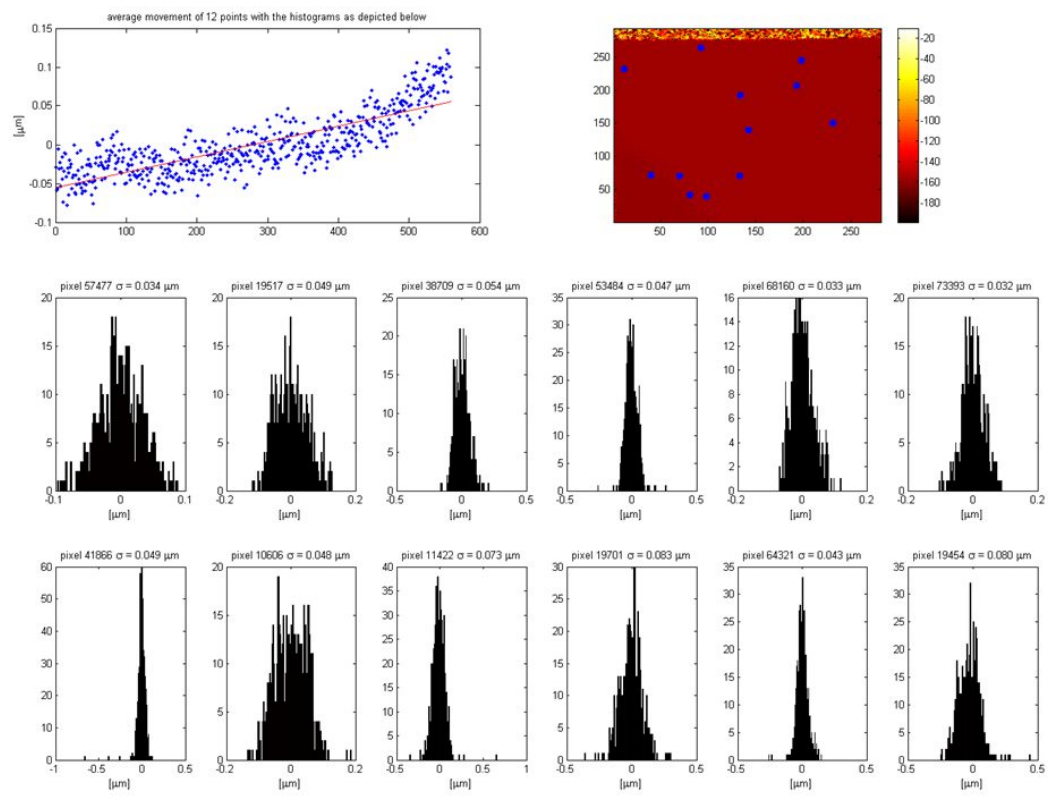

figure 6 Gage R\&R experiment results. (Top right) tracer location in field, (Top left) common mode drift, (Histograms) height variationaverage $1 \sigma=52 \mathrm{~nm}$

\section{TRACEABILITY TO STANDARDS}

For traceability to standards a method has been devised with certified calibration objects. These calibrate both the optical magnification and the axial scale of the measurement system (see Figure 7).
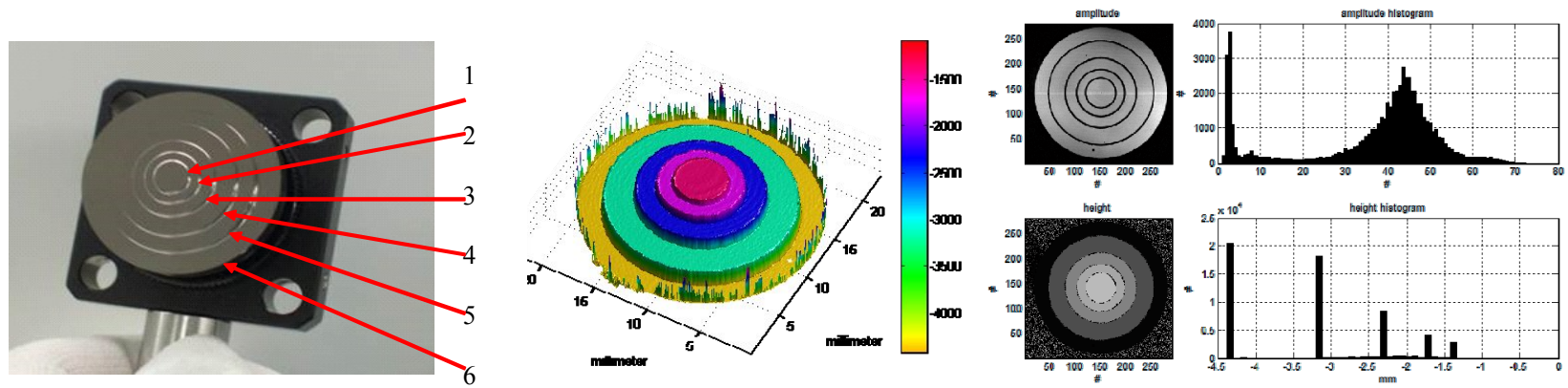

Figure 7 Calibration to international standards via a product specific transfer standard 


\section{APPLICATIONS AND FUTURE WORK}

Future developments of the sensor system will allow for higher resolution and more accurate measurements under more severe conditions. Applications of this technology are to be found in many fields, among which are inspection and metrology of carbon fiber reinforced composites, mass manufacturing of optical lenses, semiconductor defect inspection of dies; alignment of complex optical systems such as space telescopes. Next projects will implement more detailed Critical To Quality parameter extraction as well as methods to implement these in real time production physics model control loops.
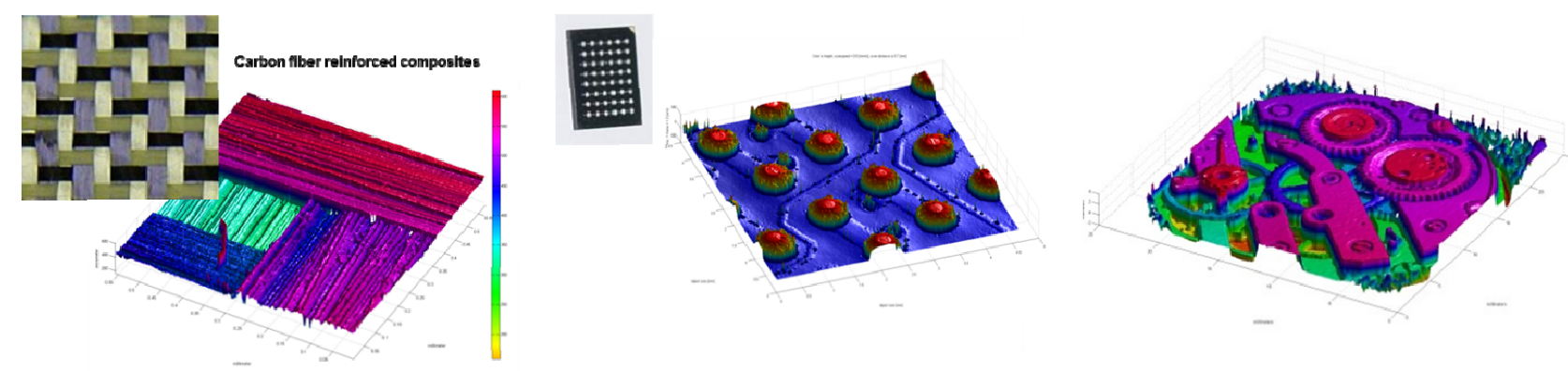

Figure 8 (left) fibers in woven Kevlar/carbon (center) high gloss plumbium balls on the black resin of a BGA chip demonstrating the high dynamic range and capturing angle of the sensor (right) complex mechanics of a wrist watch

\section{CONCLUSION}

A fast white light interferometric metrology system has been designed, built and tested by a model based design according to end user requirements. This as a means to create a Zero Defect production line. The system has undergone a successful field test of 15 month in a cold forming line of a manufacturing workshop. Accuracy and reproduction have been proven to meet with the requirements. Future work will concentrate both on improved sensors and the digital twin with digital production physics for active control of Zero Defect quality.

Acknowledgement: The work leading to these results has received funding from the European Community`s Seventh Framework Programme under grant agreement n FP7-285030

\section{REFERENCES}

[1] Richard Leach, Optical measurement of surface topography, Springer-verlag Heidelberg, 2011

[2] Bouma, Handbook of Optical Coherence Tomography, Dekker, New York, 2002

[3] Michael Bass, Handbook of optics, McGraw-Hill Education, 2009

[4] Thomas Liebig, Quality control 4.0, Microniek, Dutch Society of Precision Engineering, nr 22014

[5] Jos Havinga, Optimization and Control of Metal Forming Processes, PhD thesis University of Twente, April 2016

[6] Eugene Hecht, Optics, Addison-Wesley, 2002 